\title{
Patrones de distribución geográfica de la riqueza de especies de roedores de la tribu Oryzomyini (Rodentia: Sigmodontinae) en Sudamérica: Evaluando la importancia de los procesos de colonización y extinción
}

\author{
Geographic patterns of richness distribution of rodents species from the Oryzomyini tribe \\ (Rodentia: Sigmodontinae) in South America: Evaluating the importance of colonization \\ and extinction processes
}

\section{EVELYN VALENCIA-PACHECO ${ }^{1}$, JORGE AVARIA-LLAUTUREO ${ }^{1}$, CHRISTIAN MUÑOZ-ESCOBAR $^{1}$, DUSAN BORIC-BARGETTO ${ }^{1,2}$ \& CRISTIÁN E. HERNÁNDEZ ${ }^{1}$, *}

\author{
${ }^{1}$ Laboratorio de Diversidad Molecular y Filoinformática, Departamento de Zoología, Facultad de Ciencias Naturales y \\ Oceanográficas, Universidad de Concepción. Casilla 160-C, Concepción, Chile \\ ${ }^{2}$ Departamento de Ecología, Pontificia Universidad Católica de Chile, Alameda 340, Santiago 6513677, Chile \\ *Autor correspondiente: cristianhernand@udec.cl
}

\begin{abstract}
RESUMEN
La tribu de roedores Oryzomyini es la más diversa dentro de la subfamilia Sigmodontinae. Está constituida por 120 especies y 31 géneros, de las cuales 83 son endémicas del continente sudamericano. Este grupo exhibe una extensa distribución que abarca toda la región Neotropical, caracterizada por la presencia de una mayor riqueza en la Amazonia con una disminución monotónica hacia el sur y norte de Sudamérica. Este es un patrón bastante conocido en la mayoría de los taxones, por lo que se han propuesto varios mecanismos causales. Sin embargo, se desconocen los mecanismos que dan cuenta de este gradiente latitudinal en la riqueza de especies de Oryzomyini. Debido a que estas especies son originarias de la Amazonia, y que a través de su historia colonizaron ambientes nuevos y más variables (sur y norte), se evaluó la hipótesis fuente-sumidero, mediada por procesos de colonización y extinción, como potencial explicación al patrón de riqueza observado. Para evaluar esta hipótesis se reunió una base de datos de distribución y riqueza de especies por grado de latitud para todo el continente sudamericano. Posteriormente se evaluó el grado de anidamiento mediante los índices de T (temperatura), BR (discrepancia) y NODF. Nuestros resultados evidenciaron un grado de anidamiento significativo en la distribución de Oryzomyini, y un anidamiento significativo separadamente en filas y columnas de la matriz. Por lo tanto, se concluye que la dinámica fuente-sumidero afecta el patrón de distribución de la riqueza de roedores Oryzominos, a través de un proceso de colonización durante su expansión en Sudamérica que fue mediado por los rangos de tolerancia de las especies. Finalmente, las especies menos tolerantes se habrían extinguido, lo que determinaría un menor número de especies hacia el sur y norte de la Amazonia.
\end{abstract}

Palabras clave: anidamiento, biodiversidad, gradiente latitudinal, hipótesis fuente-sumidero, macroecología.

\begin{abstract}
The Oryzomyini rodent tribe is the most diverse taxon within the Sigmodontinae subfamily. This tribe includes 120 species and 31 genera, of which 83 are endemic to the South American continent. This tribe presents a wide distribution, covering the entire Neotropical biogeographic province, and is characterized by the presence of greater richness in the Amazonian area, with a monotonic decrease towards the south and north of South America. This pattern is wellknown in many taxa, for which various causal mechanisms have been proposed. However, the mechanisms that explain this pattern of species richness along the latitudinal gradient in the Oryzomyini tribe are unknown. Given that these species are native to Amazonia, and that throughout their history they colonized new and more variable environments (towards the south and north), we evaluated the source-sink hypothesis, mediated by processes of colonization and extinction, as a potential explanation for the observed pattern of richness. To test this hypothesis we built a database of species' distribution and richness every one degree of latitude for the entire South American continent. Subsequently we assessed the degree of nestedness using the T (temperature), BR (discrepancy) and NODF indices. Our results show a significant degree of nesting in the Oryzomyini distribution, and a significant degree of nesting of the rows and columns of the data matrix, separately. Therefore, we conclude that source-sink dynamics affect the pattern of richness distribution of Oryzomine rodents, through a process of colonization during its expansion in South America, which was conditioned by the range of tolerance of the species. This resulted in the extinction of the less tolerant species, which finally resulted in a lower number of species towards the south and north of Amazonia.
\end{abstract}

Key words: biodiversity, latitudinal gradient, macroecology, nesting, source-sink hypothesis. 


\section{INTRODUCCIÓN}

La familia Cricetidae comprende un grupo de roedores de distribución mundial, siendo la subfamilia Sigmodontinae un linaje que se diferenció de modo independiente en Sudamérica (Reig 1981, Pardiñas et al. 2002, Steppan et al. 2004). Los géneros de esta subfamilia han sido clasificados en diferentes tribus (Hershkovitz 1966, Reig 1980, Smith \& Patton 1999, D’Elía et al. 2007). De estas, Oryzomyini es la tribu más diversa dentro de la radiación de los Sigmodontinos, comprendiendo actualmente 31 géneros y 120 especies (Percequillo et al. 2011), de las cuales 83 son endémicas del continente sudamericano. Esta tribu muestra una extensa distribución geográfica, abarcando toda la región Neotropical (Weksler 2006) y se caracteriza por la presencia de una mayor riqueza en zonas de bajas latitudes (i.e., Zona Amazónica), con una disminución monotónica hacia el sur y norte de Sudamérica. Este es un patrón bastante conocido en la mayoría de los taxones (e.g., Hawkins et al. 2003, 2007, Buckley et al. 2010). Sin embargo, se desconocen los mecanismos que dan cuenta de este gradiente latitudinal en la riqueza de especies de la tribu Oryzomyini. Aunque es posible encontrar una gran variedad de estudios realizados en esta tribu con distintos propósitos, principalmente con respecto a su sistemática, taxonomía y biogeografía (e.g., Reig 1984, Gallardo \& Palma 1990, Engel et al. 1998, Pardiñas et al. 2004, Pardiñas \& Teta 2005, Weksler 2006, Weksler et al. 2006, Almeida et al. 2007, Percequillo et al. 2011). Si bien la mayoría de los estudios actuales se han centrado en esclarecer la monofilia y composición de la tribu (Voss \& Carletton 1993, Steppan 1995, Weksler 2003), relaciones filogenéticas de los nodos internos (Carletton \& Olsson 1999, Smith \& Patton 1999, Bonvicino \& Moreira 2001, Weksler 2003, 2006, Voss \& Weksler 2009), delineación de grupos de géneros (Voss \& Carletton 1993, Voss et al. 2002, Weksler et al. 2006) y descripción de fósiles (Carletton \& Olsson 1999, Pardiñas 2008, Turvey et al. 2010, Zijlstra et al. 2010), estos no han asociado la biodiversidad observada de forma explícita en un contexto geográfico. Ruggiero et al. (1998) a través del estudio de mamíferos sudamericanos, plantean la importancia de considerar la estructura biogeográfica en análisis de gradientes de distribución de especies, destacando la extensa cordillera de los Andes como estructurador de su distribución. Particularmente, para Sigmodontinos Pardiñas \& Teta (2005), analizan el efecto de las barreras geográficas, siendo la formación de los Andes de gran importancia para los procesos de especiación de estos roedores (Reig 1980, 1984, Weksler 2006).

Nosotros proponemos que, si consideramos el patrón general de disminución de la riqueza de Oryzomyini hacia el norte y sur de Sudamérica, después de su origen en la Amazonia (Weksler 2006) las especies descendientes de esta tribu dispersaron hacia latitudes con ambientes más variables, en las cuales solo las especies con mayores rangos de tolerancia sobrevivieron y las menos tolerantes se extinguieron. Esta hipótesis podría explicar los patrones de distribución de la riqueza en Oryzomyini para Sudamérica, lo cual habría generado históricamente una dinámica fuentesumidero (Pulliam 1988, Pulliam \& Danielson 1991), con las especies menos tolerantes acumulándose en latitudes cercanas al ecuador y las más tolerantes abarcando amplias distribuciones, colonizando los extremos Austral y Septentrional de Sudamérica. En esta hipótesis la población ancestral-fuente de latitudes bajas (i.e. Amazonia) habría presentado un mayor éxito reproductivo local en relación a la mortalidad local, y por lo tanto las emigraciones habrían sido mayores que las inmigraciones. Mientras que las poblaciones descendientes de otras latitudes habrían actuado como sumideros con una mayor mortalidad local e inmigración. El hecho que una población en su hábitat particular sea fuente o sumidero dependerá de las condiciones ambientales y la proximidad con otras poblaciones (Brown et al. 1996).

En la actualidad, esta dinámica ha sido aplicada en estudios de conservación, donde la identificación de hábitat fuentes, ha sido útil para determinar áreas de conservación y disminuir así la extinción de especies (e.g., Dias 1996, Novaro et al. 2000, 2005). Además, la dinámica fuente-sumidero es considerada una potencial hipótesis para explicar el cambio de la estructura poblacional y diversidad de especies (O’Keefe et al. 2009), como resultado de una dinámica de colonización y extinción (Moreno et al. 2008). Dicha dinámica, ha sido sugerida debido a que localmente las densidades poblacionales podrían permitir "exportar" emigrantes (como hábitat fuentes) y de esta 
manera colonizar otras áreas, siendo capaces de sustentar otras poblaciones o hábitat sumidero (Brown et al. 1996). Sin embargo, las especies que no poseen las capacidades necesarias para colonizar y/o establecerse en las nuevas áreas exploradas, potencialmente terminarían en un proceso de extinción.

A pesar que la dinámica fuente-sumidero ha sido considerada originalmente para metapoblaciones (Dias 1996), esta puede ser aplicada de forma análoga a metacomunidades, debido a que la capacidad de dispersión de los individuos que emigran es propia de cada especie. En consecuencia, aquellas especies con baja capacidad de dispersión tienden a estar presentes solo o casi solo en parches de hábitat sustentables (fuentes) y especies con alta vagilidad pueden frecuentemente presentarse en parches de hábitat desfavorables (sumideros) (Pulliam 2000). Hecho que en el caso de los roedores de la tribu Oyzomyini, habría permitido la colonización tanto de las zonas más australes, como septentrionales, del continente sudamericano.

Específicamente, si la diversificación de los roedores Oryzominos ocurrió desde ancestros de la Amazonia que colonizaron ambientes cada vez más variables como los australes, entonces la hipótesis fuente-sumidero explicaría su patrón actual de diversidad. De acuerdo a esta hipótesis, habría ocurrido un intercambio de individuos entre metacomunidades de la Amazonia como hábitat fuente (zona óptima) y los nuevos ambientes colonizados hacia el sur y norte de Sudamérica como hábitat sumidero (zonas subóptimas). De este modo, los ancestros de la Amazonia habrían diversificado en Sudamérica a través de un proceso de colonización y extinción, en el cual la cordillera fue uno de los principales ejes de dispersión (Reig 1984, Weksler 2006). En este proceso, especies ancestrales colonizaron nuevas áreas geográficas, ampliando sus rangos de distribución llegando a ambientes favorables o zonas óptimas (hábitat fuentes) que permitieron su persistencia, y a zonas subóptimas (hábitat sumideros) donde potencialmente se extinguieron o diversificaron por adaptación local. Por otra parte, no siempre las características de los hábitat son constantes en el tiempo y zonas óptimas podrían ser subóptimas en diferentes momentos y viceversa, lo que habría permitido la colonización de nuevos hábitat más australes en diferentes momentos de la historia de Sudamérica (e.g., periodos interglaciares).

En base a lo anteriormente expuesto, en este trabajo se evalúa la hipótesis fuente-sumidero como reflejo de los procesos de colonización y extinción que podrían explicar el patrón de riqueza de Oryzomyini en Sudamérica, hipótesis que es posible evaluar a través del grado de anidamiento en la riqueza de especies en el paisaje (Darlington 1957, Daubenmire 1975). Para poner a prueba esta hipótesis se plantearon los siguientes objetivos: (1) recopilar una base de datos de rangos de distribución latitudinal de las especies de Oryzomyini en Sudamérica; (2) cuantificar la cantidad de especies presentes en cada grado de latitud, abarcando todo el continente sudamericano; (3) determinar el grado de anidamiento en la riqueza de especies.

\section{MÉTODOS}

\section{Base de datos}

Se recopilaron los rangos de distribución de las 83 especies de la tribu Oryzomyini endémicas de Sudamérica (Tabla 1). Las especies incluidas en este listado se extienden desde los $12^{\circ} \mathrm{N}$ (península de Guajira, Colombia) a los $56^{\circ} \mathrm{S}$ (Cabo de Hornos, Chile). Un total de 40 especies fueron omitidas de la base de datos original (120 especies), debido a que sus rangos de distribución se extienden fuera de los límites de Sudamérica, y por lo tanto su historia no necesariamente está ligada a la historia del paisaje en Sudamérica. Los rangos de distribución de las especies fueron extraídos desde "The IUCN Red list of threatened species". Escogimos esta base de datos, por la presencia de los rangos de distribución para todas las especies analizadas y por la compatibilidad para ser utilizada con el software ArcMap 9.3 y de este modo determinar la riqueza de especies por banda latitudinal. Se determinó el extremo norte y sur de su distribución latitudinal asumiendo una distribución continua entre estos extremos (Tabla 1), para luego cuantificar la riqueza de especies por grado de latitud contando la intersección de las áreas de distribución por banda latitudinal en el programa ArcMap 9.3, implementado en el programa ArcGis 9.3.

\section{Evaluación de la hipótesis fuente-sumidero}

Para evaluar la hipótesis planteada se realizó un análisis de anidamiento (Darlington 1957, Daubenmire 1975), el cual evalúa de forma robusta la dinámica fuentesumidero (Moreno et al. 2008), considerando a sitios con pequeños ensambles como un subconjunto de otras áreas con un mayor número de especies (Darlington 1957, Atmar \& Patterson 1993). Esta aproximación metodológica corresponde a un tipo de prueba indirecta en la evaluación de hipótesis biogeográficas en un contexto histórico-geográfico, donde la dinámica 


\section{TABLA 1}

Listado de especies y rangos de distribución latitudinal.

Species list and latitudinal distribution ranges.

\begin{tabular}{|c|c|}
\hline Especie & Distribución latitudinal \\
\hline \multicolumn{2}{|l|}{ Aegialomys } \\
\hline A. xanthaeolus (Thomas, 1894) & $56^{\circ} \mathrm{S}-11^{\circ} \mathrm{N}$ \\
\hline \multicolumn{2}{|l|}{ Amphinectomys } \\
\hline A. savamis Malygin, 1994 & $14^{\circ} \mathrm{S}-13^{\circ} \mathrm{S}$ \\
\hline \multicolumn{2}{|l|}{ Cerradomys } \\
\hline C. andersoni (Brooks, Baker, Vargas, Tarifa, Aranibar \& Rojas, 2004) & $13^{\circ} \mathrm{S}$ \\
\hline C. maracajuensis (Langguth \& Bonvicino, 2002) & $39^{\circ} \mathrm{S}-10^{\circ} \mathrm{N}$ \\
\hline C. marinhus (Bonvicino, 2003) & $18^{\circ} \mathrm{S}-15^{\circ} \mathrm{S}$ \\
\hline C. scotti (Langguth \& Bonvicino, 2002) & $53^{\circ} \mathrm{S}-11^{\circ} \mathrm{N}$ \\
\hline C. subflavus (Wagner, 1842) & $34^{\circ} \mathrm{S}-10^{\circ} \mathrm{N}$ \\
\hline \multicolumn{2}{|l|}{ Eremoryzomys } \\
\hline E. polius (Osgood, 1913) & $33^{\circ} \mathrm{S}-21^{\circ} \mathrm{N}$ \\
\hline \multicolumn{2}{|l|}{ Euryoryzomys } \\
\hline E. emmonsae (Musser, Carleton, Brothers \& Gardner, 1998) & $26^{\circ} \mathrm{S}-19^{\circ} \mathrm{S}$ \\
\hline E. lamia (Thomas, 1901) & $35^{\circ} \mathrm{S}-21^{\circ} \mathrm{S}$ \\
\hline E. legatus (Thomas, 1925) & $34^{\circ} \mathrm{S}-21^{\circ} \mathrm{S}$ \\
\hline E. macconnelli (Thomas, 1910) & $33^{\circ} \mathrm{S}-10^{\circ} \mathrm{N}$ \\
\hline E. nitidus (Thomas, 1884) & $35^{\circ} \mathrm{S}-10^{\circ} \mathrm{N}$ \\
\hline E. russatus (Wagner, 1848) & $31^{\circ} \mathrm{S}-8^{\circ} \mathrm{N}$ \\
\hline \multicolumn{2}{|l|}{ Handleyomys } \\
\hline H. fuscatus (J.A. Allen, 1912) & $25^{\circ} \mathrm{S}-4^{\circ} \mathrm{N}$ \\
\hline H. intectus (Thomas, 1921) & $18^{\circ} \mathrm{S}-14^{\circ} \mathrm{S}$ \\
\hline \multicolumn{2}{|l|}{ Holochilus } \\
\hline H. brasiliensis (Desmarest, 1819) & $23^{\circ} \mathrm{S}-9^{\circ} \mathrm{N}$ \\
\hline H. chacarius Thomas, 1906 & $25^{\circ} \mathrm{S}-0^{\circ}$ \\
\hline H. sciureus Wagner, 1842 & $35^{\circ} \mathrm{S}-10^{\circ} \mathrm{N}$ \\
\hline \multicolumn{2}{|l|}{ Hylaeamys } \\
\hline H. acritus (Emmons \& Patton, 2005) & $9^{\circ} \mathrm{S}-5^{\circ} \mathrm{S}$ \\
\hline H. laticeps (Lund, 1840) & $7^{\circ} \mathrm{S}-6^{\circ} \mathrm{S}$ \\
\hline H. perenensis (J.A. Allen, 1901) & $24^{\circ} \mathrm{S}-4^{\circ} \mathrm{S}$ \\
\hline H. tatei (Musser, Carleton, Brothers \& Gardner, 1998) & $12^{\circ} \mathrm{S}$ \\
\hline H. yunganus (Thomas, 1902) & $32^{\circ} \mathrm{S}-7^{\circ} \mathrm{N}$ \\
\hline H. oniscus (Thomas, 1904) & $16^{\circ} \mathrm{S}-2^{\circ} \mathrm{S}$ \\
\hline \multicolumn{2}{|l|}{ Lundomys } \\
\hline L. molitor (Winge, 1887) & $10^{\circ} \mathrm{S}-3^{\circ} \mathrm{S}$ \\
\hline \multicolumn{2}{|l|}{ Melanomys } \\
\hline M. robustulus Thomas, 1914 & $10^{\circ} \mathrm{S}-8^{\circ} \mathrm{S}$ \\
\hline M. zunigae (Sanborn, 1949) & $8^{\circ} \mathrm{S}$ \\
\hline \multicolumn{2}{|l|}{ Microryzomys } \\
\hline M. altissimus (Osgood, 1933) & $18^{\circ} \mathrm{S}-4^{\circ} \mathrm{S}$ \\
\hline M. minutus (Tomes, 1860) & $22^{\circ} \mathrm{S}-7^{\circ} \mathrm{N}$ \\
\hline \multicolumn{2}{|l|}{ Mindomys } \\
\hline M. hammondi (Thomas, 1913) & $4^{\circ} \mathrm{S}-3^{\circ} \mathrm{S}$ \\
\hline \multicolumn{2}{|l|}{ Neacomys } \\
\hline N. dubosti Voss, Lunde \& Simmons, 2001 & $6^{\circ} \mathrm{S}-2^{\circ} \mathrm{S}$ \\
\hline N. guianae Thomas, 1905 & $5^{\circ} \mathrm{S}-1^{\circ} \mathrm{S}$ \\
\hline N. minutus Patton, da Silva \& Malcolm, 2000 & $13^{\circ} \mathrm{S}-10^{\circ} \mathrm{N}$ \\
\hline N. musseri Patton, da Silva \& Malcolm, 2000 & $18^{\circ} \mathrm{S}-11^{\circ} \mathrm{N}$ \\
\hline N. paracou Voss, Lunde \& Simmons, 2001 & $30^{\circ} \mathrm{S}-9^{\circ} \mathrm{S}$ \\
\hline N. spinosus (Thomas, 1882) & $30^{\circ} \mathrm{S}-8^{\circ} \mathrm{N}$ \\
\hline N. tenuipes Thomas, 1900 & $25^{\circ} \mathrm{S}-12^{\circ} \mathrm{N}$ \\
\hline
\end{tabular}


TABLA 1. Continuación

\begin{tabular}{|c|c|}
\hline Especie & Distribución latitudinal \\
\hline \multicolumn{2}{|l|}{ Nectomys } \\
\hline N. rattus (Pelzen, 1883) & $32^{\circ} \mathrm{S}-7^{\circ} \mathrm{N}$ \\
\hline N. squamipes (Brants, 1827) & $17^{\circ} \mathrm{S}-10^{\circ} \mathrm{N}$ \\
\hline N. apicalis Peters, 1861 & $25^{\circ} \mathrm{S}-11^{\circ} \mathrm{N}$ \\
\hline N. magdalenae Thomas, 1897 & $22^{\circ} \mathrm{S}-13^{\circ} \mathrm{S}$ \\
\hline \multicolumn{2}{|l|}{ Nephelomys } \\
\hline N. auriventer (Thomas, 1890) & $16^{\circ} \mathrm{S}-1^{\circ} \mathrm{N}$ \\
\hline N. caracolus (Thomas, 1914) & $14^{\circ} \mathrm{S}$ \\
\hline N. keaysi (J.A. Allen, 1900) & $14^{\circ} \mathrm{S}-0^{\circ}$ \\
\hline N. levipes (Thomas, 1902) & $18^{\circ} \mathrm{S}-6^{\circ} \mathrm{N}$ \\
\hline N. meridensis (Thomas, 1894) & $13^{\circ} \mathrm{S}-10^{\circ} \mathrm{S}$ \\
\hline \multicolumn{2}{|l|}{ Oecomys } \\
\hline O. auyantepui Tate, 1939 & $27^{\circ} \mathrm{S}-9^{\circ} \mathrm{S}$ \\
\hline O. catherinae Thomas, 1909 & $29^{\circ} \mathrm{S}-3^{\circ} \mathrm{N}$ \\
\hline O. cleberi Locks, 1981 & $10^{\circ} \mathrm{S}-0^{\circ}$ \\
\hline O. concolor (Wagner, 1845) & $22^{\circ} \mathrm{S}-7^{\circ} \mathrm{S}$ \\
\hline O. flavicans (Thomas, 1894) & $24^{\circ} \mathrm{S}-13^{\circ} \mathrm{S}$ \\
\hline O. mamorae (Thomas, 1906) & $25^{\circ} \mathrm{S}-13^{\circ} \mathrm{S}$ \\
\hline O. paricola (Thomas, 1904) & $25^{\circ} \mathrm{S}-13^{\circ} \mathrm{S}$ \\
\hline O. phaeotis (Thomas, 1901) & $22^{\circ} \mathrm{S}-1^{\circ} \mathrm{S}$ \\
\hline O. rex Thomas, 1910 & $13^{\circ} \mathrm{S}-4^{\circ} \mathrm{S}$ \\
\hline O. roberti (Thomas, 1904) & $15^{\circ} \mathrm{S}-0^{\circ}$ \\
\hline O. rutilus Anthony, 1921 & $3^{\circ} \mathrm{S}-5^{\circ} \mathrm{S}$ \\
\hline O. superans Thomas, 1911 & $17^{\circ} \mathrm{S}-6^{\circ} \mathrm{N}$ \\
\hline \multicolumn{2}{|l|}{ Oligoryzomys } \\
\hline O. andinus (Osgood, 1914) & $16^{\circ} \mathrm{S}-1^{\circ} \mathrm{S}$ \\
\hline O. arenalis (Thomas, 1913) & $18^{\circ} \mathrm{S}-10^{\circ} \mathrm{S}$ \\
\hline O. brendae Massoia, 1998 & $18^{\circ} \mathrm{S}$ \\
\hline O. chacoensis (Myers \& Carleton, 1981) & $27^{\circ} \mathrm{S}-6^{\circ} \mathrm{N}$ \\
\hline O. delticola (Thomas, 1917) & $22^{\circ} \mathrm{S}-19^{\circ} \mathrm{S}$ \\
\hline O. destructor (Tschudi, 1844) & $25^{\circ} \mathrm{S}-6^{\circ} \mathrm{S}$ \\
\hline O. eliurus (Wagner, 1845) & $22^{\circ} \mathrm{S}-7^{\circ} \mathrm{S}$ \\
\hline O. flavescens (Waterhouse, 1837) & $24^{\circ} \mathrm{S}-1^{\circ} \mathrm{S}$ \\
\hline O. fornesi (Massoia, 1973) & $13^{\circ} \mathrm{S}-0^{\circ}$ \\
\hline O. griseolus (Osgood, 1912) & $10^{\circ} \mathrm{S}-1^{\circ} \mathrm{S}$ \\
\hline O. longicaudatus (Bennett, 1832) & $13^{\circ} \mathrm{S}-8^{\circ} \mathrm{S}$ \\
\hline O. magellanicus (Bennett, 1836) & $13^{\circ} \mathrm{S}-12^{\circ} \mathrm{S}$ \\
\hline O. microtis (Allen, 1916) & $26^{\circ} \mathrm{S}-2^{\circ} \mathrm{N}$ \\
\hline O. nigripes (Olfers, 1818) & $24^{\circ} \mathrm{S}-2^{\circ} \mathrm{S}$ \\
\hline O. stramineus Bonvicino \& Weksler, 1998 & $13^{\circ} \mathrm{S}-6^{\circ} \mathrm{S}$ \\
\hline O. rupestris (Weksler \& Bonvicino, 2005) & $7^{\circ} \mathrm{S}$ \\
\hline O. moojeni (Weksler \& Bonvicino, 2005) & $22^{\circ} \mathrm{S}-7^{\circ} \mathrm{S}$ \\
\hline \multicolumn{2}{|l|}{ Oreoryzomys } \\
\hline O. balneator (Thomas, 1900) & $13^{\circ} \mathrm{S}-12^{\circ} \mathrm{S}$ \\
\hline \multicolumn{2}{|l|}{ Oryzomys } \\
\hline O. gorgasi Hershkowitz, 1971 & $13^{\circ} \mathrm{S}-12^{\circ} \mathrm{S}$ \\
\hline \multicolumn{2}{|l|}{ Pseudoryzomys } \\
\hline P. simplex (Winge, 1887) & $19^{\circ} \mathrm{S}-3^{\circ} \mathrm{S}$ \\
\hline \multicolumn{2}{|l|}{ Scolomys } \\
\hline S. melanops Anthony, 1924 & $9^{\circ} \mathrm{S}-7^{\circ} \mathrm{S}$ \\
\hline S. ucayalensis Pacheco, 1991 & $6^{\circ} \mathrm{S}-5^{\circ} \mathrm{S}$ \\
\hline \multicolumn{2}{|l|}{ Sooretamys } \\
\hline S. angouya (Fischer, 1814) & $13^{\circ} \mathrm{S}-4^{\circ} \mathrm{S}$ \\
\hline \multicolumn{2}{|l|}{ Zygodontomys } \\
\hline Z. brunneus Thomas, 1898 & $7^{\circ} \mathrm{S}-4^{\circ} \mathrm{S}$ \\
\hline
\end{tabular}


metacomunitaria es analizada en un sistema espacial jerarquizado o anidado, incorporando de forma implícita un componente temporal al considerar grandes escalas espaciales (Holling 1992, Allen et al. 2006). Un sistema anidado dará como resultado patrones de distribución no aleatorios (Connor \& Simberloff 1979, 1986, Jackson et al. 1992), donde especies raras -en términos de ocurrencia- solo estarán presentes en los ensambles con mayor riqueza, mientras que las más comunes estarán presentes en todos los sitios (Ulrich et al. 2009). Lo anterior se originaría por una relación jerárquica entre las especies y su susceptibilidad a la extinción (Patterson \& Atmar 1986, Bruun \& Moen 2003, Wethered \& Lawes 2005), su capacidad de colonización (Patterson 1990, Cook \& Quinn 1995, Honnay et al. 1999, McAbendroth et al. 2005), o su sensibilidad en función a filtros ambientales (Greve et al. 2005, Driscoll 2008), determinando así la presencia de estas en las regiones o unidades biogeográficas (Cutler 1998).

Como primer paso para evaluar esta hipótesis, se construyó una matriz de presencia-ausencia de especies, donde las columnas representan las bandas latitudinales y las filas las especies presentes en ellas. Posteriormente, se procedió a ordenar la matriz de acuerdo a la sumatoria total por filas y columnas, donde las especies comunes fueron situadas en las filas superiores y las bandas más ricas en especies fueron situadas en las columnas de la izquierda. Cuando los datos son organizados de esta forma, el anidamiento es expresado como una concentración de presencias en la esquina superior izquierda de la matriz (Atmar \& Patterson 1993, Ulrich et al. 2009). Para estimar el grado de anidamiento en la matriz, se utilizó el índice de "temperatura" T (Atmar \& Patterson 1993), el índice de discrepancia (BR) (Brualdi \& Sanderson 1999) y el índice NODF (Almeida-Neto et al. 2008). El primer índice está basado en hipótesis biogeográficas (i.e. biogeografía de islas), donde ausencias en lugares predominantemente ocupadas de la matriz y a su vez presencias en sitios predominantemente desocupadas, son menos probables (Ulrich et al. 2009), el índice T cuantifica la suma de cuadrados de distancia euclidiana de las ausencias y presencias inesperadas por sobre y debajo la isoclina respectivamente, que demarca un anidamiento perfecto (Rodríguez-Gironés \& Santamaría 2006), este índice varía en una escala de cero a 100, indicando un mayor grado de anidamiento a menores valores. Por otra parte, el índice BR compara y mide la diferencia (i.e. discrepancia) de una matriz analizada con respecto a una matriz perfectamente anidada, considerando un número mínimo de reemplazos de presencias y/o ausencias entre filas y columnas para obtener un anidamiento perfecto. Al igual que el índice $\mathrm{T}$, a menores valores de $\mathrm{BR}$, mayor será el grado de anidamiento. Finalmente el índice NODF, que a diferencia de los anteriores, además de estimar el anidamiento general de la matriz, permite calcularlo de forma independiente entre columnas y filas, evaluando el grado de anidamiento solo entre sitios (i.e. composición de especies) o solo entre especies (i.e. incidencia de especies), respectivamente. Este índice varía en una escala de cero a 100, donde a valores mayores de NODF indican un aumento en el grado de anidamiento.

Como segundo paso, se evaluó la significancia estadística de los índices calculados (i.e. T, BR y NODF), a través de la generación de un modelo nulo a partir del algoritmo de Monte Carlo, contrastando los valores observados con una distribución de probabilidad aleatoria. En nuestro caso, para datos binarios, utilizamos un modelo nulo con filas fijas y columnas equiprobables, donde los totales observados por filas son mantenidos, pero los totales de las columnas varían aleatoriamente (Patterson \& Atmar 1986, Gotelli 2000), este modelo nulo conserva la frecuencia de ocurrencia de especies y permite que la riqueza específica varíe equiprobablemente entre bandas latitudinales. Se seleccionó este modelo de bandas equiprobables considerando que a priori todas las bandas latitudinales podrían ser ocupadas por las especies y no existe un sesgo particular dado por la geografía (modelo nulo). Sin embargo, la frecuencia de ocurrencia de las especies en el paisaje es dependiente de la biología de estas, y por tanto para las especies no se puede asumir que tienen igual probabilidad de ocupar espacios, consecuentemente el modelo usado utiliza la ocurrencia de las especies como fija y conserva la frecuencia de ocurrencia observada. Además, estudios de simulación con matrices aleatorias han demostrado que alterar la suma de las filas (i.e. incidencia de las especies) desde los valores observados puede generar que los análisis sean vulnerables al error estadístico Tipo I (Gotelli 2000). Sin embargo, cambios en la restricción asignada por el modelo a las columnas no genera este problema. Para generar la distribución de frecuencia de datos nulos, se realizó un total de 50000 iteraciones.

Finalmente, se infirió el proceso que modula o promueve el patrón de anidamiento (Kadmon 1995, Lomolino 1996, Hecnar et al. 2002, Bruun \& Moen 2003), evaluando si la estructura de subconjuntos anidados es influenciada por inmigración selectiva (i.e. colonización), extinción selectiva, o ambos. Para ello se realizaron reordenamientos de las columnas dentro de la matriz, considerando el factor área (superficie de bandas latitudinales) y aislamiento (distancia geográfica entre bandas) propuestos por Lomolino (1996) para inferir indirectamente procesos de colonización $\mathrm{y} / \mathrm{o}$ extinción (Ulrich et al. 2009). Según Lomolino (1996), si la estructura es influenciada por inmigración selectiva, la matriz debería exhibir anidamiento cuando las columnas son reordenadas en orden creciente de aislamiento desde la columna o banda de latitud más rica en especies; en cambio, si es influenciada por extinción selectiva, se debiese presentar anidamiento al reordenar las columnas en sentido decreciente por área de superficie desde la columna o banda latitudinal más rica en especies. Todos los análisis de anidamiento se realizaron en el programa NODF (Almeida-Neto \& Ulrich 2011), y para visualizar la matriz ordenada se utilizó el programa Nested Calculator (Atmar \& Patterson 1993, 1995).

\section{RESULTADOS}

La riqueza de especies mostró un marcado gradiente latitudinal, presentando una disminución del número de especies desde la Amazonia hacia el sur y norte de Sudamérica (Fig. 1). Los resultados fueron concordantes con una matriz significativamente anidada (Fig. 2), con valores de los índices menores a lo esperado por azar para el índice $\mathrm{T} y$ $\mathrm{BR}, \mathrm{y}$ mayores a lo esperado por azar en el índice NODF $(\mathrm{P}<0.0001$, Tabla 2). Además, se observa un anidamiento significativo independiente entre filas (i.e. incidencia de 


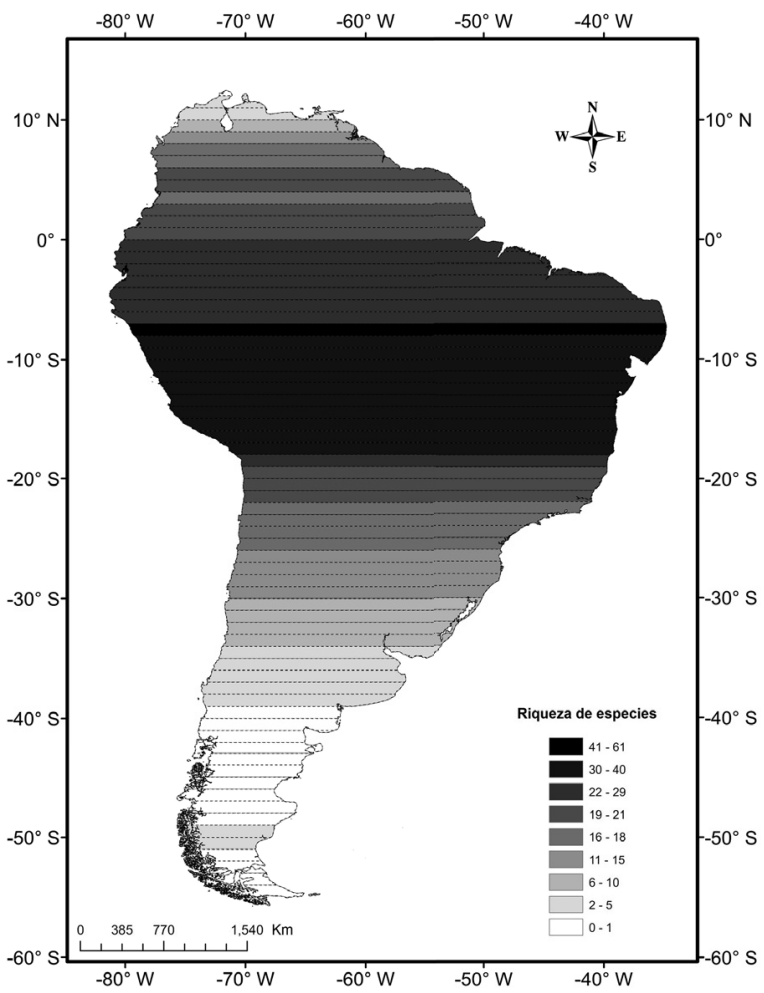

Fig. 1: Patrón geográfico latitudinal de la riqueza de especies de la tribu Oryzomyini. Cada banda corresponde a un grado de latitud.

Geographical pattern of Oryzomyini species richness. Each band corresponds to one latitudinal degree.

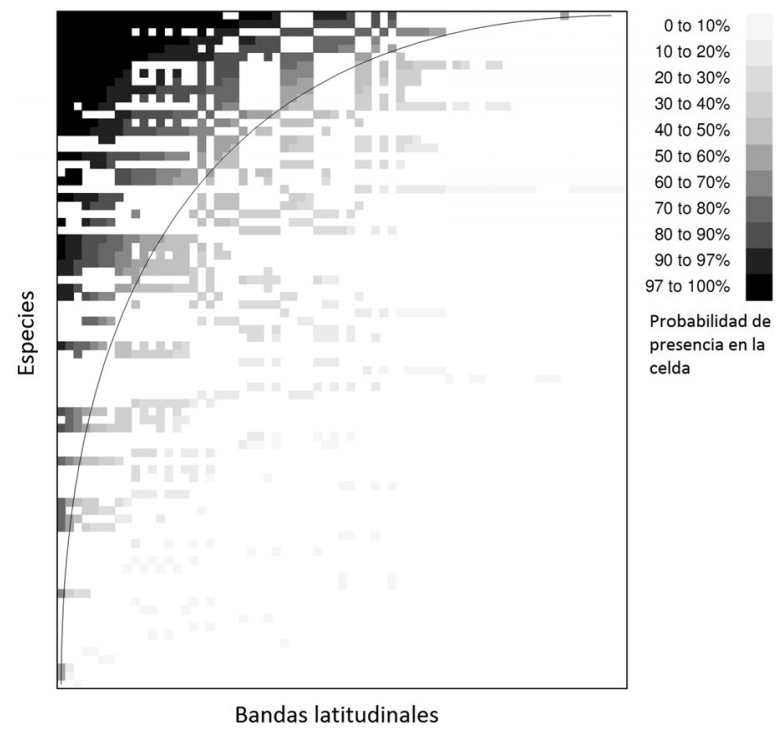

Fig. 2: Matriz de probabilidad de presencia de especies de la Tribu Oryzomyini. Las filas corresponden a las especies y las columnas son los grados de latitud en Sudamérica. La línea sólida corresponde a la isolínea que delimita el anidamiento perfecto.

Probability matrix of species state occupancy for the Oryzomyini Tribe. The rows correspond to species, and columns to the latitude in South America. The solid line shows the isoline that defines perfect nestedness. 
especies) y columnas (i.e. composición de especies) con valores del índice NODF mayores a lo esperado por azar $(\mathrm{P}<0.0001$, Tabla 2$)$. Al realizar el reordenamiento de la matriz con el objetivo de evaluar los mecanismos propuestos por Lomolino (1996), considerando un criterio de áreas de las bandas latitudinales y el grado de distancia entre estas, obtuvimos un alto grado de anidamiento $(\mathrm{P}<0.0001$, Tabla 3$)$ para los índices utilizados. Tal grado de anidamiento también se produce de forma independiente, en filas y columnas dentro de la matriz (Tabla 3 ).

\section{DISCUSIÓN}

Aún no existe un común acuerdo para definir los procesos que dieron origen a los actuales patrones de biodiversidad de los roedores Oryzominos (Weksler 2006). Esto a pesar de los notables avances en la investigación de este grupo, específicamente a través de reconstrucciones filogenéticas de algunos representantes de esta tribu (e.g., D'Elia 2000, 2003, Jansa \& Weksler 2004, Steppan et al. 2004, D'Elia et al. 2006, Weksler et al. 2006), y del descubrimiento de nuevos registros fósiles (e.g., McFarlane \& Debrot 2001, Pardiñas et al. 2002, 2003, Teta et al. 2004, Pardiñas 2008, Voss \& Weksler 2009, Turvey et al. 2010, Zijlstra et al. 2010, Percequillo et al. 2011), siendo los procesos que explican la presencia de una mayor riqueza en zonas de bajas latitudes (i.e. Zona Amazónica), con una disminución monotónica hacia el sur y norte de Sudamérica, aún desconocidos.

Nuestros resultados basados en análisis de anidamiento y modelos nulos de la ocurrencia de especies en el espacio, evidencian la existencia de un marcado patrón de anidamiento en la distribución de la riqueza de especies de la tribu Oryzomyini en Sudamérica (Tabla 2, Fig. 2). Esto sugiere que la distribución en subconjuntos de estas especies no está determinada por el azar, o bien existe un patrón de distribución no aleatorio para los ensambles de roedores de la tribu Oryzomyini en Sudamérica. En términos geográficos, sitios en altas latitudes presentarían ensambles que serían un subconjunto de especies en relación a zonas más cercanas a los trópicos (Figs. 1 y 2). Por otro lado, dado que las áreas de las bandas latitudinales y el grado de distancia entre estas estuvieron significativamente anidadas, se deduce que los procesos de colonización y extinción juegan un rol fundamental en generar el patrón de distribución de la riqueza (Tabla 3). Tal grado de anidamiento también se produce de forma independiente, en filas y columnas dentro de la matriz (Tabla 3), donde la incidencia de especies y la composición de las mismas por cada banda latitudinal se distribuyen de manera que están limitadas por su capacidad de colonización y/o su susceptibilidad a extinguirse hacia las zonas más lejanas a la Amazonia. En particular hacia zonas más australes del continente sudamericano, patrón que describe el resultado observado en la matriz de forma general (Fig. 2).

Estos resultados concuerdan con diversos estudios que revelan sistemas significativamente anidados (e.g., Atmar \& Patterson 1993, Peintinger et al. 2003, Wang et al. 2010). Sin embargo, ha existido mucho debate sobre los mecanismos subyacentes al anidamiento, como por ejemplo, si la extinción o la colonización selectiva es la causante de los patrones anidados (Ulrich et al. 2009). Al respecto, Méndez (2004) sugiere que ambos procesos se dan simultáneamente, y por consiguiente, en la práctica la colonización y extinción pueden considerarse dos caras de la misma moneda, señalando que esto es especialmente válido para casos no insulares, como el continente sudamericano. Esto último se ve reflejado en nuestros resultados, dado que el patrón de anidamiento está siendo influenciado por ambos procesos (Tabla 3), que actuarían de manera complementaria a través de la historia. Tal como sugiere Hanski (1982), quien indica que la composición de especies en muchos hábitats discontinuos o fragmentados, en los cuales el anidamiento es habitual, depende de un balance entre colonización y extinción.

En particular, nuestros resultados de un patrón de anidamiento en Oryzomyini sustenta lo esperado por la dinámica fuente-sumidero, donde los hábitat pobres en especies (i.e. sumidero) ubicados a mayores latitudes en el continente sudamericano, son un subconjunto de los más ricos (i.e. fuente) presentes a menores latitudes. En esta dinámica Dias (1996) propone que los hábitat suelen estar conectados mediante migración, de modo que los individuos originados en el hábitat 
óptimo se desplazan y colonizan el subóptimo colindante. Por consiguiente, en el caso de especies que tienen dispersión limitada (e.g., Nephelomys caracolus Thomas 1914, Oryzomys gorgasi Hershkowitz 1971) pueden frecuentemente estar ausentes en hábitat subóptimos por la dificultad de alcanzar tales áreas (Pulliam 2000). Por el contrario, una activa dispersión de las especies desde hábitat fuente podría mantener grandes poblaciones sumideros y tal dispersión podría ser evolutivamente estable (Pulliam 1988). En este sentido, acentuamos la importancia de la capacidad de dispersión de las especies, en generar la dinámica fuente-sumidero y el patrón de riqueza observado. Especialmente,

TABLA 2

Grados de anidamiento de la matriz de riqueza de la tribu Oryzomyini en Sudamérica. Se muestran los índices T, BR y NODF para la tribu Oryzomyini, y grados de anidamiento con el índice NODF por columnas y filas independientemente. (IC $95 \%$ : Intervalo de confianza al $95 \%$; *: P < 0.0001).

Degrees of nestedness for the species richness matrix of the Oryzomyini tribe of South America. The table shows the $\mathrm{T}, \mathrm{BR}$, and NODF indices for the Oryzomyini tribe, and the degree of nestedness for columns and rows, independently, using the NODF index. (IC $95 \%$ : Confidence interval $95 \%$; *: P<0.0001).

\begin{tabular}{lccccc}
\hline & Índice T & Índice BR & Índice NODF & NODF Columnas & NODF Filas \\
\hline Calculado & $15.5^{*}$ & $423^{*}$ & $37.12^{*}$ & $34.71^{*}$ & $38.78^{*}$ \\
Media esperada por azar & 35.4 & 584.29 & 25.8 & 27.68 & 24.49 \\
(IC 95\%) & $(33.2-37.6)$ & $(574-594)$ & $(25.04-26.59)$ & $(26.53-28.77)$ & $(23.88-25.19)$ \\
\hline
\end{tabular}

TABLA 3

Grados de anidamiento para la matriz de la tribu Oryzomyini reordenada por área y distancia. Se muestran los índices T, BR y NODF, respectivamente. ( $\mathrm{T}_{\mathrm{c}}$ : Índice de Temperatura calculado; $\mathrm{T}_{\mathrm{e}}$ : Índice de temperatura esperado por azar; $\mathrm{BR}_{\mathrm{c}}$ : Índice de discrepancia calculado; $\mathrm{BR}_{\mathrm{e}}$ : Índice de discrepancia esperado por azar; $\mathrm{NODF}_{\mathrm{c}}$ : Índice NODF calculado; $\mathrm{NODF}_{\mathrm{e}}$ : Índice NODF esperado por azar; IC $95 \%$ : Intervalo de confianza al $95 \%$; ${ }^{*}$ P $\left.<0.0001\right)$.

Degree of nestedness for the Oryzomyini tribe matrix re-ordered by area and distance. The table shows the T, BR, and NODF indices, respectively. ( $T_{c}$ : Temperature index calculated; $T_{e}$ : Mean temperature index expected by chance; $B R_{c}$ : Discrepancy index calculated; $\mathrm{BR}_{\mathrm{e}}$ : Mean discrepancy index expected by chance; $\mathrm{NODF}_{\mathrm{c}}$ : NODF index calculated; $\mathrm{NODF}_{\mathrm{e}}$ : Mean NODF index expected by chance; IC $95 \%$ : Confidence interval $95 \%$; * $\mathrm{P}<0.0001$ ).

\begin{tabular}{lcccccc}
\hline & \multicolumn{3}{c}{ Índice $\mathrm{T}$} \\
\hline & $\mathrm{T}_{\mathrm{c}}$ & $\mathrm{T}_{\mathrm{e}}$ & $(\mathrm{IC} 95 \%)$ & $\mathrm{BR}_{\mathrm{c}}$ & $\mathrm{BR}_{\mathrm{e}}$ & $(\mathrm{IC} 95 \%)$ \\
\hline Área & $15.77^{*}$ & 45.85 & $(42.7-49)$ & $423^{\star}$ & 584.38 & $(574-594)$ \\
Distancia & $16.42^{\star}$ & 45.85 & $(42.7-49.1)$ & $423^{\star}$ & 584.37 & $(574-594)$ \\
\hline
\end{tabular}

\begin{tabular}{llllllllll}
\hline & \multicolumn{3}{c}{ Índice NODF } & \multicolumn{3}{c}{ Filas } & \multicolumn{3}{c}{ Columnas } \\
\hline & NODF $_{\mathrm{c}}$ & $\mathrm{NODF}_{\mathrm{e}}$ & $(\mathrm{IC} 95 \%)$ & NODF $_{\mathrm{c}}$ & NODF $_{\mathrm{e}}$ & $(\mathrm{IC} \mathrm{95 \% )}$ & NODF $_{\mathrm{c}}$ & NODF $_{\mathrm{e}}$ & (IC 95 \%) \\
Área & $35.46^{*}$ & 20.15 & $(18.9-21.3)$ & $38.78^{\star}$ & 24.49 & $(23.9-25.1)$ & $30.65^{\star}$ & 13.85 & $(11.2-16.4)$ \\
Distancia & $36.4^{*}$ & 20.15 & $(18.9-21.4)$ & $38.78^{\star}$ & 24.49 & $(23.9-25.1)$ & $32.94^{\star}$ & 13.84 & $(11.2-16.5)$ \\
\hline
\end{tabular}


considerando la biología de los roedores de la tribu Oryzomyini, como organismos vágiles y donde el comportamiento exploratorio está muy desarrollado (Weksler 2006, Weksler et al. 2006, Percequillo et al. 2011). Nosotros sugerimos que desde su origen en la zona Amazónica estos roedores avanzaron hacia el sur y norte de Sudamérica colonizando nuevos hábitat de acuerdo al siguiente escenario sustentado en nuestros resultados: (1) Durante su diversificación, los ancestros de Oryzomyini fueron colonizando nuevos hábitat cada vez más variables en el sur y norte de Sudamérica desde hábitat fuente ubicados en la Amazonia, donde la Cordillera de los Andes jugó un papel fundamental en la dispersión a zonas australes (ver Reig, 1984); (2) En este proceso, algunas especies lograron adaptarse, aumentando sus tamaños poblacionales, y por difusión lograron colonizar hasta el extremo sur y norte de Sudamérica; (3) Específicamente, las nuevas condiciones australes, cada vez más variables solo permitieron la llegada de unas pocas especies con amplios rangos de tolerancia (e.g., Holochilus brasilensis Desmarest, Oligoryzomys longicaudatus Bennett), lo cual explicaría el bajo número de especies a mayores latitudes, donde solo fueron capaces de llegar y establecerse las pocas especies con mayores habilidades adaptativas. De hecho, Pardiñas et al. (2011) sugieren que en el extremo sur de Sudamérica las condiciones predominantes durante los periodos glaciales habrían producido condiciones de mayor aridez que afectaron fuertemente la presencia de especies de la tribu Oryzomyini, las cuales son reconocidas especies de ambientes húmedos (Weksler 2006). En consecuencia, una amplia zona de la Patagonia y Tierra del Fuego (Extremo Sur de Sudamérica) solo presentaría una especie de la tribu (Fig. 1), O. longicaudatus, la cual ha logrado colonizar ambientes con condiciones térmicas tan diversas como las que imperan en el extremo sur del desierto de Atacama $\left(27^{\circ}\right.$ S) o en la Patagonia llegando hasta los $55^{\circ} \mathrm{S}$ (Mann 1978, Palma et al. 2005, Belmar-Lucero et al. 2009).

Finalmente, la hipótesis fuente-sumidero es una hipótesis sustentable que puede dar cuenta de la distribución de la riqueza, no solo en Oryzomyini, sino que en diversos taxones que presentan un gradiente en su riqueza de especies en Sudamérica, escenario histórico donde los procesos de colonización y extinción jugaron un rol fundamental en determinar los patrones actuales de distribución de la biodiversidad.

AGRADECIMIENTOS: Este trabajo fue financiado por el Proyecto FONDECYT N ${ }^{\circ} 11080110$ a cargo de Cristián E. Hernández. Agradecemos a Paula Neill, Bryan MoralesPallero, y Enrique Rodríguez-Serrano por sus sugerencias y comentarios; y a Andrés Santana Mardones, por su colaboración en los análisis de datos georeferenciados. Christian Muñoz-Escobar y Dusan Boric-Bargetto agradecen el financiamiento de la Escuela de Graduados de la Universidad de Concepción en el marco del Programa de Doctorado en Sistemática y Biodiversidad, y a la beca CONICYT de Doctorado Nacional. Finalmente, Jorge Avaria-Llautureo agradece el financiamiento de la beca CONICYT de Magíster Nacional.

\section{LITERATURA CITADA}

ALLEN CR, AS GARMESTANI, TD HAVLICEK, PA MARQUET, GD PETERSON, C RESTREPO, CA STOW \& BE WEEKS (2006) Patterns in body mass distributions: Sifting among alternative hypotheses. Ecology Letters 9: 630-643.

ALMEIDA FC, CR BONVICINO \& P CORDEIROESTRELA (2007) Phylogeny and temporal diversification of Calomys (Rodentia, Sigmodontinae): Implications for the biogeography of an endemic genus of the open/dry biomes of South America. Molecular Phylogenetics and Evolution 42: 449-466.

ALMEIDA-NETO M, P GUIMARÃES, PR GUIMARÃES JR, RD LOYOLA \& W ULRICH (2008) A consistent metric for nestedness analysis in ecological systems: Reconciling concept and measurement. Oikos 117: 1227-1239.

ALMEIDA-NETO M \& W ULRICH (2011) A straightforward computational approach for measuring nestedness using quantitative matrices. Environmental Modelling \& Software 26: 173-178.

ATMAR W \& B PATTERSON (1993) The measure of order and disorder in the distribution of species in fragmented habitat. Oecologia 96: 373-382.

ATMAR W \& B PATTERSON (1995) The nestedness temperature calculator: A visual basic program including, 294 presence-absence matrices. AICS Research Inc., University Park, NM and The Field Museum, Chicago (en linea) URL: http://www. aics-research.com/nestedness/tempcalc.html (accedido Noviembre 16, 2010).

BELMAR-LUCERO S, P GODOY, M FERRÉS, P VIAL \& RE PALMA (2009) Range expansion of Oligoryzomys longicaudatus (Rodentia, Sigmodontinae) in Patagonia Chile, and first record of Hantavirus in the region. Revista Chilena de Historia Natural 82: 265-275.

BONVICINO CR \& MA MOREIRA (2001) Molecular phylogeny of the genus Oryzomys (Rodentia: Sigmodontinae) based on cytochrome b DNA sequences. Molecular Phylogenetics and Evolution 18: 282-292.

BROWN JH, GC STEVENS \& DM KAUFMAN (1996) The geographic range: Size, shape, boundaries, 
and internal structure. Annual Review of Ecology and Systematics 27: 597-623.

BRUALDI RA \& JG SANDERSON (1999) Nested species subsets, gaps, and discrepancy. Oecologia 119: 256-264.

BRUUN HH \& J MOEN (2003) Nested communities of alpine plants on isolated mountains: Relative importance of colonization and extinction. Journal of Biogeography 30: 297-303.

BUCKLEY LB, TJ DAVIES, DD ACKERLY, NJ KRAFT, SP HARRISON et al. (2010) Phylogeny, niche conservatism and the latitudinal diversity gradient in mammals. Proceedings of the Royal Society B 1-9 (en línea) URL: http:// rspb.royalsocietypublishing.org/content/ early/2010/03/18/rspb.2010.0179.full (accedido Marzo 24, 2010).

CARLETTON MD \& SL OLSON (1999) Amerigo Vespucci and the rat of Fernando de Noronha: a new genus and species of Rodentia (Muridae: Sigmodontinae) from a volcanic island off Brazil's continental shelf. American Museum Novitates 3256: $1-59$

CONNOR E \& D SIMBERLOFF (1979) The assembly of species communities: Chance or competition? Ecology 60: 1132-1140.

CONNOR E \& D SIMBERLOFF (1986) Competition, scientific method, and null hypotheses in ecology. American Scientist 74: 155-162.

COOK RR \& JF QUINN (1995) The influence of colonization in nested species subsets. Oecologia 102: 413-424.

CUTLER AH (1998) Nested patterns of species distribution: processes and implications. En: McKinney ML \& JA Drake (eds) Biodiversity dynamics: 212-231. Columbia University Press, New York.

DARLINGTON PJ (1957) Zoogeography: The geographical distribution of animals. Wiley, New York.

DAUBENMIRE R (1975) Floristic plant geography of eastern Washington and northern Idaho. Journal of Biogeography 2: 1-18.

D’ELÍA G (2000) Comments on recent advances in understanding sigmodontine phylogeny and evolution. Mastozoologia Neotropical 7: 47-54.

D’ELÍA G (2003) Phylogenetics of Sigmodontinae (Rodentia, Muroidea, Cricetidae), with special reference to the akodont group, and with additional comments on historical biogeography. Cladistics 19: 307-323.

D’ELÍA G, L LUNA, EM GONZÁLEZ \& BD PATTERSON (2006) On the Sigmodontinae radiation (Rodentia, Cricetidae): An appraisal of the phylogenetic position of Rhagomys. Molecular Phylogenetics and Evolution 38: 558-564.

D’ELÍA G, U PARDIÑAS, P TETA \& J PATTON (2007) Definition and diagnosis of a new tribe of Sigmodontine rodents (Cricetidae: Sigmodontinae), and a revised classification of the subfamily. Gayana 71: 187-194.

DIAS P (1996) Sources and sinks in population biology. Trends in Ecology and Evolution 2: 326-329.

DRISCOLL DA (2008) The frequency of metapopulations, metacommunities and nestedness in a fragmented landscape. Oikos 117: 297-309.

ENGEL SR, KM HOGAN, JF TAYLOR \& SK DAVIS (1998) Molecular Systematics and Paleobiogeography of the South American
Sigmodontine Rodents. Molecular Biology and Evolution 15: 35-49.

GALLARDO MH \& E PALMA (1990) Systematics of Oryzomys longicaudatus (Rodentia: Muridae) in Chile. Journal of Mammalogy 71: 333-342.

GOTELLI N (2000) Null model analysis of species cooccurrence patterns. Ecology 81: 2606-2621.

GREVE M, NJM GREMMEN, KJ GASTON \& SL CHOWN (2005) Nestedness of Southern Ocean island biotas: ecological perspectives on a biogeographical conundrum. Journal of Biogeography 32: 155-168.

HANSKI I (1982) Dynamics of regional distribution: the core and satellite hypothesis. Oikos 38: 210-221.

HAWKINS BA, R FIELD, HV CORNELL, DJ CURRIE, JF GUÉGAN et al. (2003) Energy, water, and broadscale geographic patterns of species richness. Ecology 84: 3105-3117.

HAWKINS BA, JAF DINIZ-FILHO, CA JARAMILLO \& SA SOELLER (2007) Climate, niche conservatism, and the global bird diversity gradient. The American Naturalist 170: 16-27.

HECNAR SJ, GS CASPER, RW RUSSELL, DR HECNAR \& JN ROBINSON (2002) Nested species assemblages of amphibians and reptiles on islands in the laurentian great lakes. Journal of Biogeography 29: 475-489.

HERSHKOVITZ P (1966) Mice, land bridges and Latin American faunal interchange. En: Wenzel RL \& VJ Tipton (eds) Ectoparasites of Panama: 725-751. Field Museum of Natural History, Chicago.

HOLLING CS (1992) Cross-scale morphology, geometry, and dynamics of ecosystems. Ecological Monographs 62: 447-502.

HONNAY O, M HERMY \& P COPPIN (1999) Nested plant communities in deciduous forest fragments: species relaxation or nested habitats? Oikos 84: $119-129$.

IUCN (2010) International Union for the Concervation of Nature and Natural Resources. IUCN Red List categories and criteria, Version 2010.4. URL: http://www.iucnredlist.org.Downloaded (accedido Octubre 27, 2010).

JACKSON DA, KM SOMERS \& HH HARVEY (1992) Null models and fish communities: evidence of nonrandom patterns. The American Naturalist 139: 930-951.

JANSA SA \& M WEKSLER (2004) Phylogeny of muroid rodents: relationships within and among major lineages as determined by IRBP gene sequences. Molecular Phylogenetics and Evolution 31: 256-276.

KADMON R (1995) Nested species subsets and geographic isolation: A case study. Ecology 76: 458-465.

LOMOLINO MV (1996) Investigating causality of nestedness of insular communities: Selective immigrations or extinctions? Journal of Biogeography 23: 699-703.

MANN GF (1978) Los pequeños mamíferos de Chile marsupiales, quirópteros, endentados $\mathrm{y}$ roedores. Ediciones Universidad de Concepción, Concepción, Chile.

MCABENDROTH L, A FOGGO, SD RUNDLE \& DT BILTON (2005) Unravelling nestedness and spatial pattern in pond assemblages. Journal of Animal Ecology 74: 41-49.

MCFARLANE DA \& AO DEBROT (2001) A new species of extinct oryzomyine rodent from the Quaternary of Curaçao, Netherlands Antilles. Caribbean Journal of Science 37: 182-184. 
MÉNDEZ M (2004) La composición de especies de aves en islas y paisajes fragmentados: Un análogo ecológico de las muñecas rusas. El Draque (España) 5: 199-212.

MORENO R, M RIVADENEIRA, CE HERNÁNDEZ, S SAMPÉRTEGUI \& N ROZBACZYLO (2008) Do Rapoport's rule, the mid-domain effect or the source-sink hypotheses predict bathymetric patterns of polychaete richness on the Pacific coast of South America? Global Ecology and Biogeography 17: 415-423.

NOVARO AJ, KH REDFORD \& RE BODMERT (2000) Effect of hunting in source-sink systems in the Neotropics. Conservation Biology 14: 713-721.

NOVARO A, M FUNES \& R WALKER (2005) An empirical test of source-sink dynamics induced by hunting. Journal of Applied Ecology 42: 910-920.

O'KEEFE K, U RAMAKRISHNAN, M VAN TUINEN \& E HADLY (2009) Source-sink dynamics structure a common montane mammal. Molecular Ecology 18: 4775-4789.

PALMA RE, R RIVERA-MILLA, J SALAZAR-BRAVO, F TORRES-PÉREZ, UFJ PARDIÑAS et al. (2005) Phylogeography of Oligoryzomys longicaudatus (Rodentia: Sigmodontinae) in temperate South America. Journal of Mammalogy 86: $191-200$.

PARDIÑAS U, G D'ELÍA \& P ORTIZ (2002) Sigmodontinos fósiles (Rodentia, Muroidea, Sigmodontinae) de América del Sur: Estado actual de su conocimiento y prospectiva. Mastozoología Neotropical 9: 209-252.

PARDIÑAS UFJ, P TETA, S CIRIGNOLI \& DH PODESTÁ (2003) Micromamíferos (Didelphimorphia y Rodentia) de norpatagonia extra andina, Argentina: Taxonomía alfa y biogeografía. Mastozoologia Neotropical 10: 69-113.

PARDIÑAS U, A ABBA \& M MERINO (2004) Micromamíferos (Didelphimorphia y Rodentia) del sudoeste de la provincia de Buenos Aires (Argentina): Taxonomía y distribución. Mastozoología Neotropical 11: 211-232.

PARDIÑAS U \& P TETA (2005) Roedores sigmodontinos del chaco húmedo de Formosa, aspectos taxonómicos y distribución geográfica. Temas de Naturaleza y Conservación (Argentina) 4: 501-517.

PARDIÑAS UFJ (2008) A new genus of Oryzomyini rodent (Cricetidae: Sigmodontinae) from the Pleistocene of Argentina. Journal of Mammalogy 89: $1270-1278$.

PARDIÑAS UFJ, P TETA, G D’ELÍA \& EP LESSA (2011) The evolutionary history of sigmodontine rodents in Patagonia and Tierra del Fuego. Biological Journal of the Linnean Society 103: 495-513.

PATTERSON BD \& W ATMAR (1986) Nested subsets and the structure of insular mammalian faunas and archipelagos. En: Heaney LR \& BD Patterson (eds) Island biogeography of mammals: 65-82. Academic Press and Linnean Society, London.

PATTERSON BD (1990) On the temporal development of nested subset patterns of species composition. Oikos 59: 330-342.

PEINTINGER M, A BERGAMINI \& B SCHMID (2003) Species-area relationships and nestedness of four taxonomic groups in fragmented wetlands. Basic and Applied Ecology 4: 385-394.

PERCEQUILLO AR, M WEKSLER \& LP COSTA (2011) A new genus and species of rodent from the Brazilian Atlantic Forest (Rodentia: Cricetidae: Sigmodontinae: Oryzomyini), with comments on
Oryzomyine biogeography. Zoological Journal of the Linnean Society 161: 357-390.

PULLIAM HR (1988) Sources, sinks, and population regulation. The American Naturalist 132: 652-661.

PULLIAM HR \& BJ DANIELSON (1991) Sources, sinks, and habitat selection: A landscape perspective on population dynamics. The American Naturalist 137: 50-66.

PULLIAM HR (2000) On the relationship between niche and distribution. Ecology Letters 3: 349-361.

REIG OA (1980) A new fossil genus of South American cricetid rodents allied to Wiedomys, with an assessment of the Sigmodontinae. Journal of Zoology 192: 257-281.

REIG OA (1981) A refreshed unorthodox view of paleobiogeography of South American Mammals (Review of G. G. Simpson, 1980. Splendid Isolation. The curious history of South American Mammals. Yale, University Press, New Haven and London). Evolution 35: 1032-1035.

REIG OA (1984) Distribuição geogrãfica e historia evolutiva dos roedores muroideos sulamericanos (Cricetidae: Sigmodontinae). Brazilian Journal of Genetics 7: 333-365.

RODRÍGUEZ-GIRONÉS MA \& L SANTAMARÍA (2006) A new algorithm to calculate the nestedness temperature of presence-absence matrices. Journal of Biogeography 33: 924-935.

RUGGIERO A, JH LAWTON \& TB BLACKBURN (1998) The geographic ranges of mammalian species in South America: Spatial patterns in environmental resistance and anisotropy. Journal of Biogeography 25: 1093-1103.

SMITH MF \& JL PATTON (1999) Phylogenetic relationships and the radiation of Sigmodontine rodents in South America: Evidence from cytochrome b. Journal of Mammalian Evolution 6: 89-128.

STEPPAN SJ (1995) Revision of tribe Phyllotini (Rodentia: Sigmodontinae), with a phylogenetic hypothesis for the Sigmodontinae. Fieldiana, Zoology 80: 1-112.

STEPPAN SJ, RM ADKINS \& J ANDERSON (2004) Phylogeny and divergence-date estimates of rapid radiations in Muroid rodents based on multiple nuclear genes. Systematic Biology 53: 533-553.

TETA P, D LOPONTE \& A ACOSTA (2004) Sigmodontinos (Mammalia, Rodentia) del Holoceno tardío del nordeste de la provincia de Buenos Aires (Argentina). Mastozoología Neotropical 11: 69-80.

TURVEY ST, $M$ WEKSLER, EL MORRIS \& M NOKKERT (2010) Taxonomy, phylogeny and diversity of the extinct Lesser Antillean rice rats (Sigmodontinae: Oryzomyini), with description of a new genus and species. Zoological Journal of the Linnean Society 160: 748-772.

ULRICH W, M ALMEIDA-NETO \& N GOTELLI (2009) A consumer's guide to nestedness analysis. Oikos 118: 3-17.

VOSS RS \& MD CARLETTON (1993) A new genus for Hesperomys molitor Winge and Holochilus magnus Hershkovitz (Mammalia, Muridae), with an analysis of its phylogenetic relationships. American Museum Novitates 3085: 1-39.

VOSS RS, M GÓMEZ-LAVERDE \& V PACHECO (2002) A new genus for Aepeomys fuscatus Allen, 1912, and Oryzomys intectus Thomas, 1921: Enigmatic murid from Andean Cloud Forests. American Museum Novitates 3373: 1-42. 
VOSS RS \& M WEKSLER (2009) On the taxonomic status of Oryzomys curasoae McFarlane and Debrot 2001, with remarks on the phylogenetic relationships of $O$. gorgasi Hershkovitz, 1971. Caribbean Journal of Science 45: 73-79.

WANG Y, Y BAO, M YU, G XU \& P DING (2010) Nestedness for different reasons: The distributions of birds, lizards and small mammals on islands of an inundated lake. Diversity and Distribution 16: 862-873.

WEKSLER M (2003) Phylogeny of Neotropical oryzomyine rodents (Muridae: Sigmodontinae) based on the nuclear IRBP exon. Molecular Phylogenetics and Evolution 29: 331-349.

WEKSLER M (2006) Phylogenetic relationships Oryzomyini rodents (Muroidea: Sigmodontinae):

Editor Asociado: Marco A. Méndez

Recibido el 26 de enero de 2011; aceptado el 27 de mayo de 2011
Separate and combined analyses of morphological and molecular data. Bulletin of the American Museum of Natural History 296: 1-149.

WEKSLER M, R PERCEQUILLO \& R VOSS (2006) Ten New Genera of Oryzomyine Rodents (Cricetidae: Sigmodontinae). American Museum Novitates 3537: 1-29.

WETHERED R \& MJ LAWES (2005) Nestedness of bird assemblages in fragmented Afromontane forest: The effect of plantation forestry in the matrix. Biological Conservation 123: 125-137.

ZILJSTRA JS, PA MADERN \& LW VAN DEN HOEK OSTENDE (2010) New genus and two new species of Pleistocene oryzomyines (Cricetidae: Sigmodontinae) from Bonaire, Netherlands Antilles. Journal of Mammalogy 91: 860-873. 
